\title{
Analyzing Borders Between Partially Contradicting Fuzzy Classification Rules
}

\author{
Andreas Nürnberger, Aljoscha Klose, Rudolf Kruse \\ University of Magdeburg, Faculty of Computer Science \\ 39106 Magdeburg, Germany \\ E-mail: andreas.nuernberger@cs.uni-magdeburg.de \\ Fax: +49 39167 12018, Phone: +49 3916711358
}

\begin{abstract}
Fuzzy classification rules allow the definition of readable and interpretable rule bases. Nevertheless, the shape of the resulting class borders of fuzzy classification rules depends to a great part on the used $t$ norm and t-conorm and can sometimes even be counter-intuitive. In this paper we discuss the shape of class borders between overlapping rules under consideration of different t-norms and t-conorms and the effect of rule aggregation, i.e. more than one rule defining the same class are overlapping. Furthermore, we discuss the influence of rule weights and point out some aspects of the classification behavior of naive Bayes classifiers, which can be seen as a subset of fuzzy systems. Our main goal is to give the potential user an insight into the classification behavior of fuzzy classifiers. For this, mainly $2 D$ and $3 D$ visualizations are used to illustrate the cluster shapes and the borders between distinct classes.
\end{abstract}

\section{Introduction}

Fuzzy classification rules are widely considered a well-suited representation of classification knowledge, as they allow readable and interpretable fuzzy rule bases [3]. Due to their abstraction from numbers to linguistic variables they resemble the way, humans would possibly formulate their knowledge [5]. Everybody can easily be convinced that the world is inherently fuzzy and that crisp thresholds can almost never be justified. Therefore, the use of fuzzy rules seems quite intuitive. Specifying fuzzy sets may seem easier than the specification of crisp intervals. One may hope that the inference system will 'somehow' deal with the uncertainties. Especially, when rule bases are automatically or half-automatically created from example data, this can hold true. But finally, when the derived classifier is applied to data, usually a winner takes all principle is used to determine a single class to which the applied data is most similar. Therefore, there are always crisp borders between regions of different predicted classes.

In [9] we had discussed the shape of these borders for two overlapping classes in two- and threedimensional universes and we had given general hints concerning the shape of higher dimensional clusters. Furthermore, we had investigated different influences that should be considered, when a fuzzy rule base is created (manually or automatically). These are, for example, the t-norm and t-conorm used, the use of threshold membership values, the type of fuzzy sets used, and the number and complexity of fuzzy rules used to define the classifier.

In this paper we discuss the shape of class borders for overlapping and aggregating rules (i.e. more than one rule defining the same class are overlapping) under consideration of the aspects mentioned above. Furthermore, we give a formula description of the shape of the class borders and we extend our discussion to the influence of rule weights, which are used in a number of fuzzy classification systems. Based on these considerations, we also point out some aspects of the classification behavior of naive Bayes classifiers, since they can be seen as a subset of fuzzy systems, as we have shown in [8].

However, our main goal is to give the potential user an intuition how different t-norms/t-conorms influences the classification behavior of fuzzy classifiers.

First, we describe the general structure of fuzzy classification systems, and how they can be derived and modified. In the subsequent sections, we use 2D and 3D visualizations to illustrate the shapes of borders between distinct classes.

\subsection{Fuzzy classification systems}

The basic idea of fuzzy classification systems [3] is to describe the supposed clusters - to which the respective classes are assigned - by vague cluster prototypes. These prototypes are defined by a number of fuzzy sets which circumscribe the cluster in each con- 
sidered dimension of the universe. Thus, a specific cluster $i$ is defined by a fuzzy classification rule of the form:

if $\quad x_{1}$ is $\mu_{i, 1}$ and $x_{2}$ is $\mu_{i, 2}$ and $\ldots x_{n}$ is $\mu_{i, n}$

then pattern $\left(x_{1}, x_{2}, \ldots, x_{n}\right)$ belongs to class $c_{k}$,

where $\mu_{i, j}$ is a membership function that defines the fuzzy set describing the cluster $i$ in dimension $j$. (In the following we use for simplification the fuzzy sets $(A$, $B, \ldots)$ instead of their membership functions $\mu$ to define fuzzy rules). A rule base defined by a number of fuzzy rules can be used to predict the class for an arbitrary pattern $\left(x_{1}, x_{2}, \ldots, x_{n}\right)$ in data space. For this, an inference scheme for the evaluation of the rule base has to be defined.

\subsection{Fuzzy inference}

For the evaluation of a fuzzy (classification) rule a t-norm has to be defined, by which the activation of the rule and thus the membership value of the pattern to the specific class $c_{k}$ can be derived. Common t-norms used for fuzzy classification systems are minimum $\left(\min ; \mathrm{T}_{\min }(a, b)=\min \{a, b\}\right)$ and algebraic product (prod; $\left.\mathrm{T}_{\text {prod }}(a, b)=a \cdot b\right)$ [5]. Furthermore, if more than one rule is used to predict the same class a t-conorm has to be defined. Usually the max t-conorm $\left(\perp_{\min }(a, b)\right.$ $=\max \{a, b\})$ is used, i.e. the highest rule activation is chosen. Further t-conorms are, e.g. prod t-conorm $\left(\perp_{\text {prod }}(a, b)=a+b-a \cdot b\right)$ or the Lukasiewicz t-conorm $\left(\perp_{\text {Luka }}(a, b)=\min \{a+b, 1\}\right)$. An example of the classification behavior of different t-conorms - resulting in a different selection of the 'winner' - for overlapping clusters is given in Table 1.

Table 1. Inference using different t-conorms ('winning' class bold)

\begin{tabular}{|l|l|l|l|}
\hline \multirow{2}{*}{ rule } & \multicolumn{3}{|l|}{ rule activation } \\
\cline { 2 - 4 } & class 1 & class 2 & class 3 \\
\hline 1 & 0.4 & 0.0 & 0.0 \\
\hline 2 & 0.4 & 0.0 & 0.0 \\
\hline 3 & 0.0 & 0.6 & 0.0 \\
\hline 4 & 0.4 & 0.0 & 0.0 \\
\hline 5 & 0.0 & 0.0 & 0.2 \\
\hline$\perp_{\text {min }}$ & 0.4 & $\mathbf{0 . 6}$ & 0.2 \\
\hline$\perp_{\text {Luka }}$ & $\mathbf{1 . 0}$ & 0.6 & 0.2 \\
\hline$\perp_{\text {prod }}$ & $\mathbf{0 . 7 8 4}$ & 0.6 & 0.2 \\
\hline
\end{tabular}

\subsection{Obtaining fuzzy classifiers}

Conventional classifiers are usually derived from data and are not specified manually, due to their (mostly) complex mathematical description. In contrast to this, fuzzy classifiers can easily be defined manually and learnt classifiers can more easily be interpreted. The most common methods to induce fuzzy classifiers from data are fuzzy clustering and neuro-fuzzy learning.

Fuzzy clustering searches for clusters in the input data (see, for example, [2][3]). The number of clusters is determined by an evaluation measure, and the size and shape of the clusters is given by the clustering algorithm. The obtained clusters can be transformed into fuzzy rules by projection of the clusters onto the individual dimensions [4].

Neuro-fuzzy learning combines fuzzy classifiers with neural networks (see, for example, [6]). These approaches create fuzzy classifiers from data by heuristic learning procedures. Usually the neuro-fuzzy learning procedures underlie certain restrictions, such that both, acceptable performance and interpretable rules can be obtained [7].

In the following we have a closer look at the classification behavior of fuzzy systems. For simplification, we consider only rule bases which are defined by use of piecewise linear fuzzy sets (e.g. triangular or trapezoidal fuzzy sets).

\section{Classifiers in a 2D data space}

An example of a simple fuzzy classification system consisting of the two fuzzy rules

If $x$ is $A_{1}$ and $y$ is $B_{1}$ then pattern $(x, y)$ belongs to $c_{1}$ If $x$ is $A_{2}$ and $y$ is $B_{2}$ then pattern $(x, y)$ belongs to $c_{2}$ where the fuzzy sets $A_{i}$ and $B_{i}$ are defined by triangular membership functions is depicted in Figure 1. The pyramids $R_{1}$ and $R_{2}$ represent the membership degrees of each data point $(x, y)$ to the respective classes $c_{i}$, obtained by use of the min t-norm.

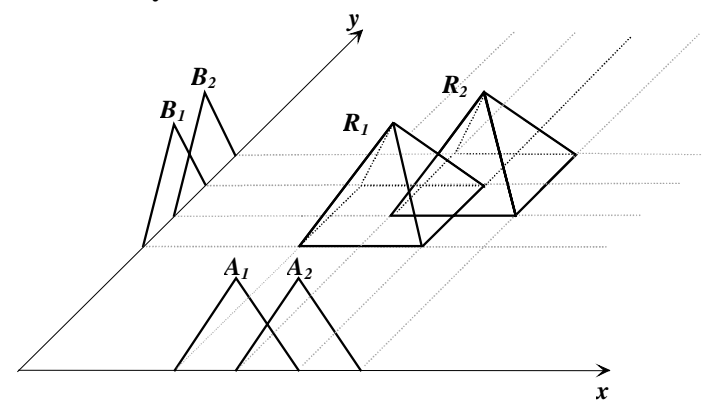

Figure 1. Representation of two fuzzy rules

\subsection{Influence of different t-norms}

The shape of the border between the two classes depends on the used t-norm as depicted in Figure 2. The border line between the two rules is defined by $\operatorname{activation}\left(R_{1}\right)=\operatorname{activation}\left(R_{2}\right)$. 
Therefore, for the $\min \mathrm{t}$-norm it must hold

$\min \left\{x\right.$ is $A_{1}, y$ is $\left.B_{1}\right\}=\min \left\{x\right.$ is $A_{2}, y$ is $\left.B_{2}\right\}$.

Since the fuzzy sets $A_{\mathrm{i}}$ and $B_{\mathrm{i}}$ are defined by piecewise linear functions (here, triangular fuzzy sets) we obtain for every area in data space in which all antecedents are linear (i.e. a cell of the grid spanned by the begin and end of every linear function used to define the included fuzzy sets)

$$
\min \left\{a_{1} x+b_{1}, c_{1} y+d_{1}\right\}=\min \left\{a_{2} x+b_{2}, c_{2} y+d_{2}\right\} .(2)
$$

This leads to a linear equation for every possible case (e.g. two diagonal and one horizontal lines in the example depicted in Figure 2a).

If the prod t-norm is used,

$$
A_{1}(x) \cdot B_{1}(y)=A_{2}(x) \cdot B_{2}(y)
$$

must hold, so we obtain (for every area in data space in which all antecedents are linear)

$$
\left(a_{1} x+b_{1}\right) \cdot\left(c_{1} y+d_{1}\right)=\left(a_{2} x+b_{2}\right) \cdot\left(c_{2} y+d_{2}\right)
$$

and thus

$$
\begin{gathered}
x y\left(a_{1} c_{1}-a_{2} c_{2}\right)+x\left(a_{1} d_{1}-a_{2} d_{2}\right)+ \\
y\left(b_{1} c_{1}-b_{2} c_{2}\right)+b_{1} d_{1}-b_{2} d_{2}=0
\end{gathered}
$$

with $a^{\prime}:=-\left(a_{1} d_{1}-a_{2} d_{2}\right), b^{\prime}:=-\left(b_{1} d_{1}-b_{2} d_{2}\right), c^{\prime}:=\left(b_{1} c_{1}\right.$ $\left.-b_{2} c_{2}\right)$ and $d^{\prime}:=\left(a_{1} c_{1}-a_{2} c_{2}\right)$ this can be rewritten as

$$
y=\frac{a^{\prime} x+b^{\prime}}{c^{\prime} x+d^{\prime}} .
$$

This equation defines a hyperbola. According to the interval in which the specific linear function (here, shoulder of the triangular) of the fuzzy sets $A_{\mathrm{i}}$ and $B_{\mathrm{i}}$ are defined, this equation specifies a section of the separation line between the classes (see Figure 2b).
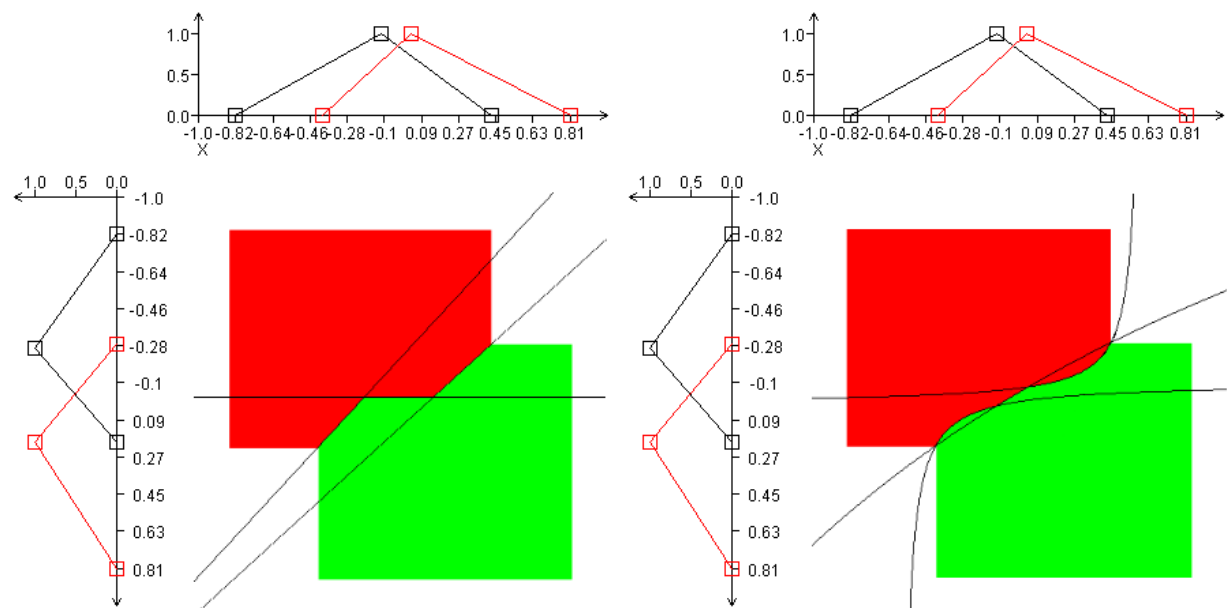

(a)

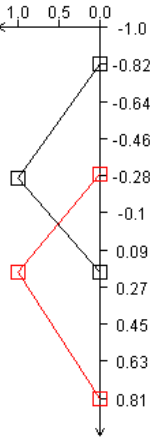

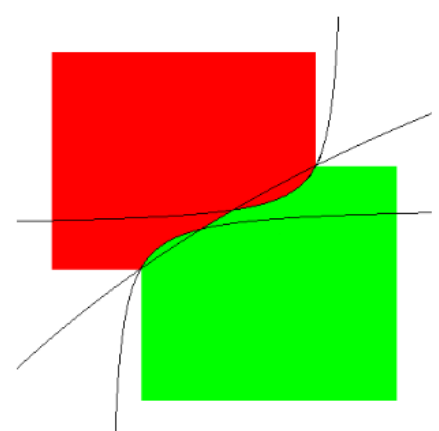

(b)

Figure 2. Evaluation of a fuzzy rule base and the resulting separation lines (extended line plotted) using tnorms $\min (\mathrm{a})$ and prod (b)
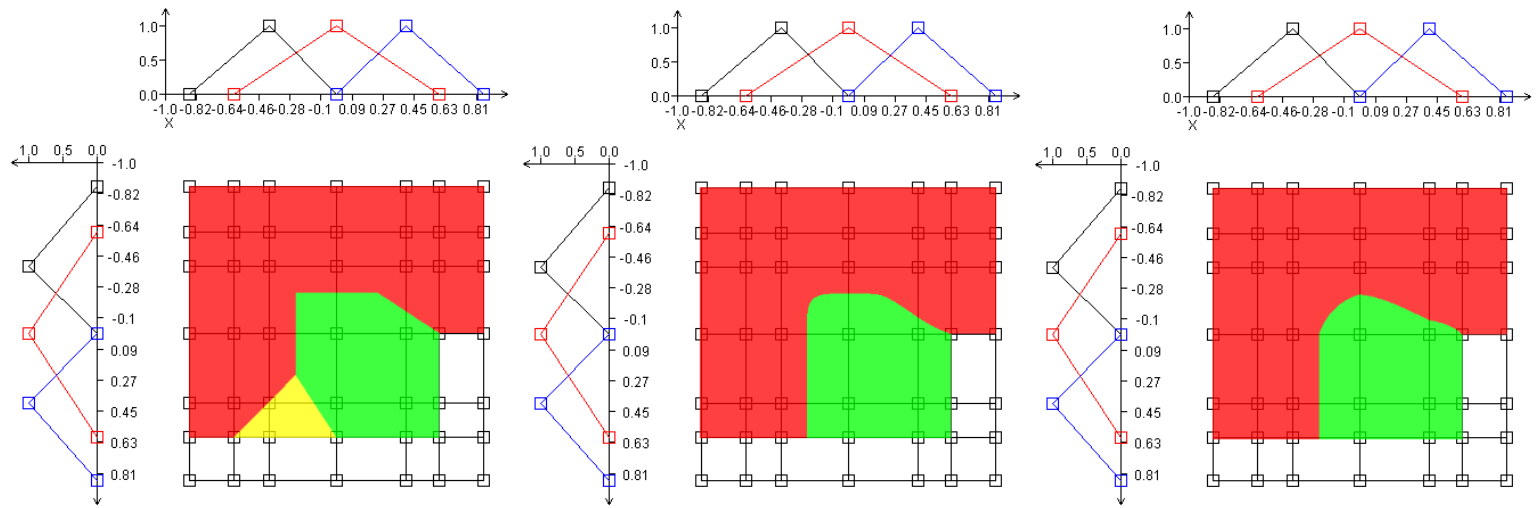

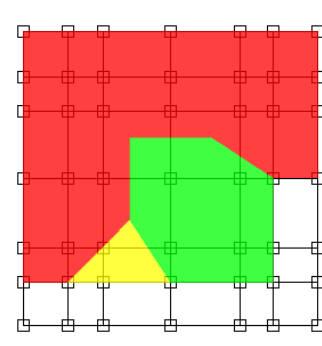

(a)

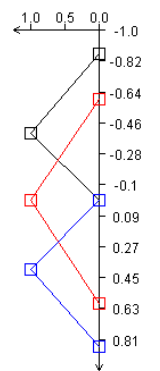

申

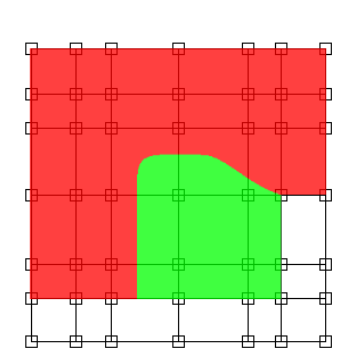

(b)

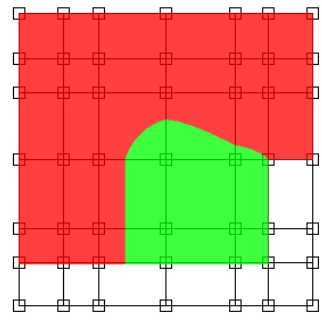

(c)

Figure 3. ,Take over' effects for overlapping clusters using t-norms $\min (\mathrm{a})$, prod (c) and an ,intermediate“ t-norm (b). The figures are obtained by use of a t-norm presented by Schweitzer in [10]. 


\subsection{Aggregation of rules}

If more than one rule is used to define one class the activations of all rules defining this class have to be aggregated to a unique class activation by use of a tconorm (see also 1.2). This can lead to unexpected effects as presented in Figure 3. For the presented figures we used a parameterized t-norm and t-conorm, which converges to the prod or min t-norm according to the used value for the parameter $p$ (see [10]):

$$
\begin{gathered}
\mathrm{T}_{\text {Schweizer }}(a, b)=\max \left(0, a^{p}+b^{p}-1\right)^{\frac{1}{p}} \\
\perp_{\text {Schweizer }}(a, b)=1-\max \left(0,(1-a)^{p}+(1-b)^{p}-1\right)^{\frac{1}{p}}
\end{gathered}
$$

The inner class (light color) is defined by the inner fuzzy sets. Although, we expect a larger area which should be assigned to this class (because of the large coverage of the inner fuzzy sets), its area in the overlapping regions is taken over to a great part by the outer class (dark color). Furthermore, the area for the inner class is reduced even more, if we use the prod $\mathrm{t}$ norm (smoothed edges). (The very light triangle in Figure 3a depicts an ambiguous area [9].)

This effect is even worse, if we define a class by a single rule which is surrounded by rules defining a different class as depicted in Figure 4 . The light area in the center depicts the area we obtain if we use $\mathrm{prod} / \mathrm{sum}$ inference. We can increase the size of this region by use of rule weights, resulting in areas depicted by the dark colored circular lines. In this way we may improve the interpretability of the obtained classifier for this specific case. Nevertheless, in most cases the use of rule weights is critical [6]. Here, we may use the rule weight to represent an (intuitively) assumed uniform distribution.

Fuzzy classifiers and probability theory. It is interesting to compare fuzzy classification rules and classifiers based on probability theory (e.g. naive bayes classifiers [1]). In probability theory independent dimensions are combined using the product, which is also very common in fuzzy based classification. The most apparent difference is the normalization of the probability densities to integrate to one in every dimension. Normalized fuzzy sets, however, are defined to have at least one point with a membership value of one, but may have any area below the curve. This leads to a considerably higher hyper volume under a rule if, for example, an antecedent is pruned, since all values of the corresponding attribute are considered as possible and therefore the membership degree is one for all possible values. The hyper volume in the probabilistic case remains one by definition. We assume balanced class frequencies and a uniform distribution over all rules defining the same class. Thus, the density of a rule is decreased, when its influence spreads.

Nevertheless, naive bayes classifiers can be mapped to (neuro-)fuzzy classifiers, when rule weights are used [8]. In this case, the rule weights are used to normalize fuzzy rules in a probabilistic sense. The area defined by the outer circular line in Figure 4 is computed according to the assumptions of a naive bayes classifier.

Overlapping of more than two classes. The classification behavior of fuzzy classifiers when more than two classes overlap can usually be transferred to the overlapping of two classes.
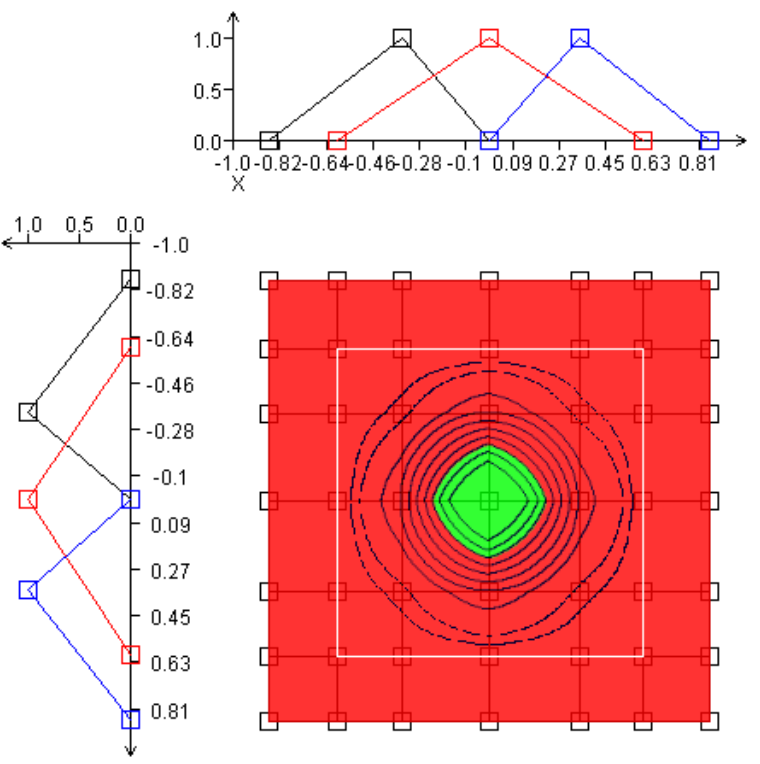

Figure 4. Use of rule weigths for every rule to relax take over effects of overlapping clusters (prod tnorm used).

\section{Classifiers in $3 D$ data space}

To obtain usable diagrams of three dimensional fuzzy clusters we omit the (direct) representation of the membership degrees. The clusters were shown by illuminated plots of the outer cluster surfaces in the 3D data space. To improve the evidence of the description, the plots are rotated. Nevertheless, all single clusters created by fuzzy classifiers are spanned by the fuzzy sets in the antecedents of the rule. Therefore, the axes of the spanning system are always parallel to the axes of the data space.

The shapes of the class borders can be derived - in the same way as presented for the two-dimensional case - for every area in data space in which all antecedents are linear. For the min t-norm we obtain hyperplane sections (see also equations (1) and (2)). For the prod t-norm (see also equations (3) to (6)) it must hold 


$$
\begin{aligned}
& \left(a_{1} x+b_{1}\right) \cdot\left(c_{1} y+d_{1}\right) \cdot\left(e_{1} z+f_{1}\right)= \\
& \left(a_{2} x+b_{2}\right) \cdot\left(c_{2} y+d_{2}\right) \cdot\left(e_{2} z+f_{2}\right) .
\end{aligned}
$$

Therefore, we obtain third order surfaces of the form

$$
\begin{gathered}
x y z a_{1}+x y a_{2}+x z a_{3}+y z a_{4}+ \\
x a_{5}+y a_{6}+z a_{7}+a_{8}=0 .
\end{gathered}
$$

Nevertheless, the class borders in any plane parallel to the axes of the coordinate system are still defined by hyperbola sections, since we just replace the respective factor of the considered dimension in equation (7) by a constant and thus obtaining an equation similar to (6). An example of the separation border of two symmetrical overlapping hypercubes is depicted in Figure 5.

In Figure 6 the 'take over' effects for a class defined by a single rule (hidden in the figure) surrounded by rules for a different class are depicted. The effects are similar to the two dimensional case (see also Figure 3 ).

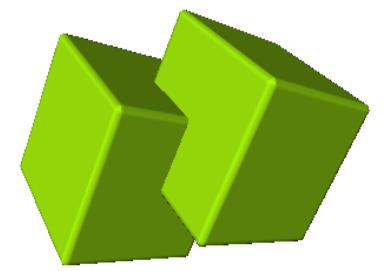

(a)

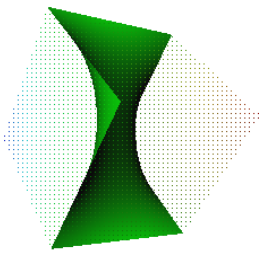

(b)
Figure 5. Two symmetrical overlapping clusters (a) and the shape of the separation border in the overlapping area (b)

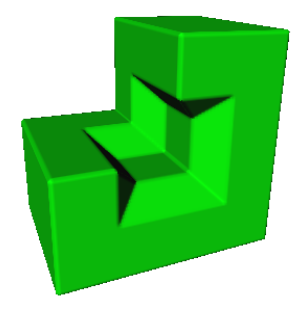

(a)

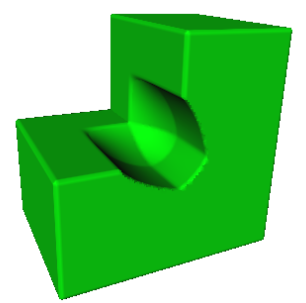

(b)
Figure 6. Symmetrical overlapping clusters $10 \mathrm{cu}$ bes of class a surrounding one cube of class $b$ : $\mathrm{min} / \mathrm{max}$ inference (a) and prod/sum inference (b)

\section{Conlusions}

In this paper we have discussed the shape of class borders for overlapping and aggregating rules under consideration of different t-norm and t-conorms and we have given a formula description of the shape of the class borders for rules using fuzzy sets defined by piecewise linear functions. Furthermore, we have shown, that in some cases the classification behavior of fuzzy classifiers can sometimes even be counterintuitive. Therefore, in some cases rule weights might be an appropriate method to improve the interpretability of the obtained classifier. In these cases, the rule weights might be used to represent an (intuitively) assumed distribution of the data. Nevertheless, rule weights might even destroy the interpretability of a fuzzy system if they are used without a (formal) justification.

Some of the tools that we used to analyze the classification behavior of fuzzy classifiers can be freely obtained from our web server (http://fuzzy.cs.unimagdeburg.de).

\section{References}

[1] I. Good, The Estimation of Probabilities: An Essay on Modern Bayesian Methods, MIT Press, Cambridge, MA, USA, 1965.

[2] D. Gustafson, and W. Kessel, Fuzzy Clustering with a Fuzzy Covariance Matrix, In: Proc. IEEE CDC, pp. 761-766, San Diego, CA, 1979.

[3] F. Höppner, F. Klawonn, R. Kruse, and T. Runkler, Fuzzy Cluster Analysis, Wiley, Chichester, 1999.

[4] F. Klawonn, and R. Kruse, Constructing a Fuzzy Controller from Data, Fuzzy Sets and Systems, 85, pp. 177-193, 1997.

[5] R. Kruse, J. Gebhardt, and F. Klawonn, Foundations of Fuzzy Systems, Wiley, 1994.

[6] D. Nauck, F. Klawonn, and R. Kruse, Foundations of Neuro-Fuzzy Systems, Wiley, Chichester, 1997.

[7] D. Nauck, A. Nürnberger, and R. Kruse, NeuroFuzzy Classification, In: Advances in Data Science and Classification, Proc. of the 6th Conference of the International Federation of Classification Societies (IFCS-98), pp. 287-294, Springer-Verlag, Berlin, 1998.

[8] A. Nürnberger, C. Borgelt, and A. Klose, Improving Naive Bayes Classifiers Using NeuroFuzzy Learning, In: Proc. 6th International Conference on Neural Information Processing '99 (ICONIP'99), Perth, Australia, pp. 154-159, IEEE, Piscataway, NJ, 1999.

[9] A. Nürnberger, A. Klose, and R. Kruse, Discussing Cluster Shapes of Fuzzy Classifiers, In: Proc. 18th International Conference of the North American Fuzzy Information Processing Society (NAFIPS'99), pp. 546-550, New York, 1999.

[10] B. Schweizer, and A. Sklar, Associative functions and abstract semigroups, Publ. Math. Debrecen., 10, pp. 69-81, 1963. 